\title{
The Lunar X-ray Observatory (LXO)/Magnetosheath Explorer in X-rays (MagEX)
}

\author{
M.R. Collier*, T.F. Abbey ${ }^{\dagger}$, N.P. Bannister ${ }^{\dagger}$, J.A. Carter ${ }^{\dagger}$, M. Choi ${ }^{*}$, T.

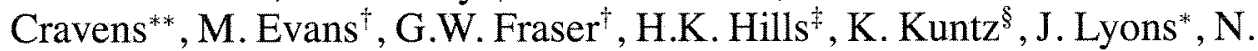 \\ Omidi $^{\ddagger}$, F.S. Porter*, A.M. Read ${ }^{\dagger}$, I. Robertson**, P. Rozmarynowski* , S. \\ Sembay $^{\dagger}$, D.G. Sibeck* ${ }^{*}$, S.L. Snowden* ${ }^{*}$ T. Stubbs ${ }^{\|}$and P. Travnicek ${ }^{\dagger \dagger}$ \\ ${ }^{*}$ NASA/GSFC, Greenbelt, MD 20771 \\ University of Leicester, UK \\ ${ }^{* *}$ University of Kansas \\ Perot Systems Government Services \\ $\S$ Johns Hopkins University, Baltimore, MD 21218 \\ ${ }^{I}$ Solana Scientific \\ $\|_{\text {Goddard Earth Science and Technology Center, UMBC }}$ \\ ${ }^{\dagger}$ Academy of Science Czech Republic
}

\begin{abstract}
X-ray observations of solar wind charge exchange (SWCX) emission, a nuisance to astrophysicists, will dramatically enhance our ability to determine the structure and variability of the Earth's magnetosheath. Such observations could be made from the lunar surface or an Earth-orbiting spacecraft and will resolve key controversies about magnetopause physics as well as better characterize SWCX emission with the aim of avoiding or removing it from astrophysical observations.
\end{abstract}

\section{SWCX EMISSION AND MAGNETOSHEATH IMAGING}

In 1996, a ROSAT observation of comet Hyakutake revealed unexpected X-ray emission originating from the vicinity of the comet [4]. Following similar observations from other comets, Cravens [2] and Cox [1] explained these observations as SWCX emission in which high charge state solar wind ions exchange electrons with cometary neutral atoms. The solar wind ions end up in excited states which relax, causing the emission of soft Xrays, as illustrated by Figure 1. Cravens et al. then found that the solar wind flux and the ROSAT soft X-ray rates were strongly correlated [3], showing that soft X-ray detectors can monitor the flux and composition of the solar wind remotely.

This early work soon led to the realization that soft X-ray imaging could probe the Earth's magnetopause which separates two distinctly different plasma regions: the shocked solar wind (magnetosheath) and magnetospheric plasma. The magnetopause boundary prevents all but a small fraction of the mass, energy, and momentum carried by the solar wind from entering the magnetosphere. However, the solar wind mass, energy and momentum that does enter the magnetosphere powers all major plasma processes within the magnetosphere. Usually, this energy is stored in the magnetosphere. 


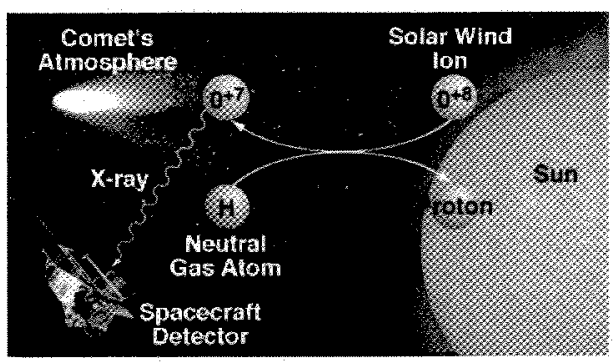

FIGURE 1. Schematic showing the process of solar wind charge exchange emission.

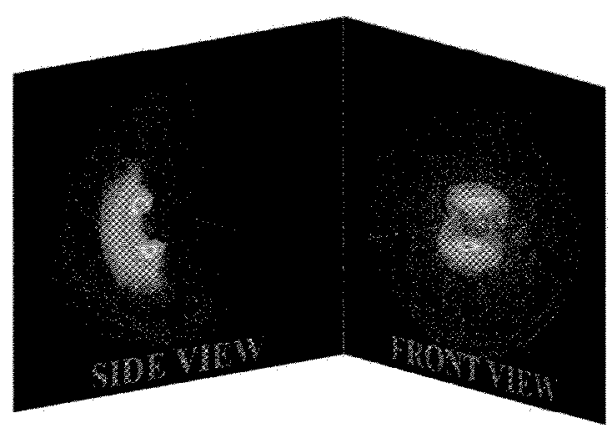

FIGURE 2. Simulations of SWCX soft X-ray emission from the terrestrial magnetosheath

However, under certain geomagnetically active conditions it can be impulsively released into the Earth's atmosphere. Thus, global imaging of the magnetosheath, the boundary between the solar wind and the magnetosphere, has the potential to revolutionize SunEarth connection studies. Furthermore, magnetopause location, shape, position, transfer processes, boundary layers, and reconnection serve as important inputs for operational space weather models.

Recent work ([7], [6]) has shown that soft X-rays emitted from the magnetosheath during both average and strong solar wind periods have sufficient flux to be observable. Figure 2 shows the side and front views of a simulation during storm time of Earth's magnetosheath. The bright red is the cusp region, an area of open field lines which allows solar wind plasma easy access to the near-Earth environment. These simulations show that the magnetosheath emission contributes significantly to the soft X-ray sky and can, at times, contribute more than all the other components put together.

\section{HELIOPHYSICS SCIENCE}

The magnetosheath region is highly heterogeneous and dynamic, as shown in the hybrid particle simulations of, for example, Omidi et al. [5]. The origin of many structures observed on the magnetopause has been controversial. Although in-situ spacecraft 


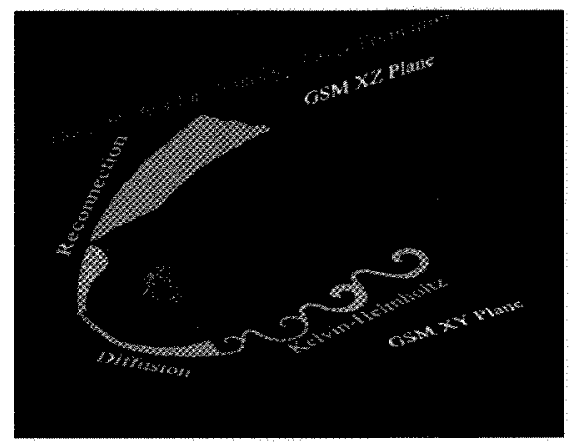

FIGURE 3. Three models of boundary layer formation

observations of the magnetosheath provide transient localized glimpses of its complex structure, its response to variable solar wind conditions, and its superimposed kinetic features, only simultaneous global observations can resolve and define the characteristics of various phenomena. We describe in this paper a global soft X-ray magnetosheath imager called the Lunar X-ray Observatory (LXO) or Magnetosheath Explorer in Xrays (MagEX) designed to be deployed on the lunar surface by astronauts. Some of the controversies which such a magnetosheath imager could resolve include:

Flux Ropes and Pressure Pulses - In situ observations of the dayside magnetopause indicate the frequent occurrence of transient $(\sim 1 \mathrm{~min})$ magnetic field perturbations. The inferred dimensions of these "flux transfer events" or FTEs are typically on the order of 1-3 $R_{E}$. Some events may be flux ropes produced by bursty reconnection along one or more reconnection lines, while others may be large amplitude waves driven by magnetosheath density variations. $\mathrm{LXO} / \mathrm{MagEX}$ will determine:

Question 1a - Are flux transfer events flux ropes or surface waves?

Question $1 \mathrm{~b}$ - How significant are flux transfer events both spatially (i.e., their dimensions) and temporally (i.e., their recurrence rates)?

Magnetic Merging/Reconnection or Boundary Layer Dynamo - A layer of magnetosheath plasma is almost always present just earthward of the magnetopause. The layer may result from diffusion (smooth profile, thickness broadens downstream), reconnection (abrupt edges, thickness broadens away from reconnection site), or mixing driven by nonlinear Kelvin-Helmholtz boundary waves (structured layer on the magnetospheric flanks). LXO/MagEX will determine:

Question 2 - Where and when do magnetic reconnection, diffusion, and the KelvinHelmholtz instability operate on the magnetopause?

The Depletion Layer - Shocked magnetosheath magnetic field lines drape against the magnetopause. Plasma flows out the ends of the field lines, resulting in a depletion layer of magnetosheath plasma with depressed densities and enhanced magnetic field 
strengths lying just outside the magnetopause. Figure 3 illustrates these models of boundary layer formation. LXO/MagEX will answer:

Question $3 \mathrm{a}$ - What solar wind conditions favor the formation of a depletion layer outside the dayside magnetopause?

Steady reconnection cannot occur in superAlfvénic flows. Nevertheless, it has been reported at high latitudes where flows are superAlfvénic. LXO/MagEX will address the question:

Question 3b - Does the subsonic transition necessary for steady-state reconnection poleward of the cusp result from a depletion layer extending to high latitudes or a deceleration of the magnetosheath flow in the vicinity of the cusp?

Slow Mode Transition or Exogenous Solar Wind Fluctuations - The bow shock, a standing fast mode wave, slows down and diverts the solar wind flow around the magnetosphere. By analogy to this wave, some researchers invoke encounters with a slow mode wave standing in the inner magnetosheath to explain transient density enhancements. Other researchers attribute these enhancements to solar wind features advecting antisunward. LXO/MagEX will answer:

Question 4 - Is the magnetosheath flow diverted around the magnetosphere gradually, abruptly by a standing slow mode wave, or via some other unidentified process?

Resolving these controversies will represent a major advance in Sun-Earth connection studies and benefit our space weather prediction capability. Furthermore, SWCX soft Xray emission will provide information on the solar wind composition and charge states.

\section{THE MAGEX/LXO MAGNETOSHEATH IMAGER}

The MagEX/LXO instrument concept is shown in Figure 4. It features slumped microchannel plate optics, a large area $\mathrm{CCD}$, an onboard power system, and passive cooling. It consists of a $25 \mathrm{~cm}$ focal length "lobster-eye" optic using an array of $4 \mathrm{~cm} \mathrm{x} 4 \mathrm{~cm}$ nickel coated square pore microchannel plates slumped to a $50 \mathrm{~cm}$ radius. This optical configuration yields a very large field-of-view, $30^{\circ}$ in this example. A flat plane of four $6 \mathrm{~cm} \times 6 \mathrm{~cm}$ CCDs is placed at the focus to provide both imaging and moderate spectral resolution.

The effective area is about $12 \mathrm{~cm}^{2}$ at $500 \mathrm{eV}$ with a point spread function of less than four arc minutes, an anticipated energy resolution of about 50 at $500 \mathrm{eV}$, a full-field grasp of $3.4 \mathrm{~cm}^{2} \mathrm{sr}$, and a $3 \times 3$ degree grasp of $0.04 \mathrm{~cm}^{2} \mathrm{sr}$.

The instrument will charge the battery during the day using solar power and only operate at night. During the day, a cover, perhaps bimetallically activated, will protect the instrument optics.

The entire package weighs about $31 \mathrm{~kg}$. This includes about $20 \mathrm{~kg}$ for the Lithion battery, about $8 \mathrm{~kg}$ for the box, $<1 \mathrm{~kg}$ for the radiator and $<2 \mathrm{~kg}$ for the electronics.

Total power consumption is estimated at $11 \mathrm{~W}$ during operation which includes $6.7 \mathrm{~W}$ 


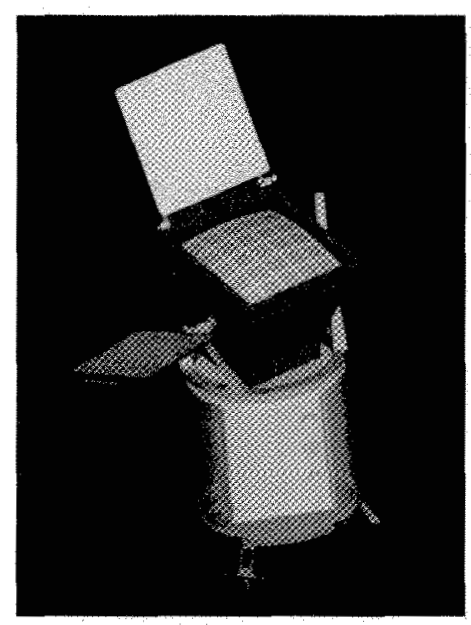

FIGURE 4. MagEX/LXO concept with Lithion battery

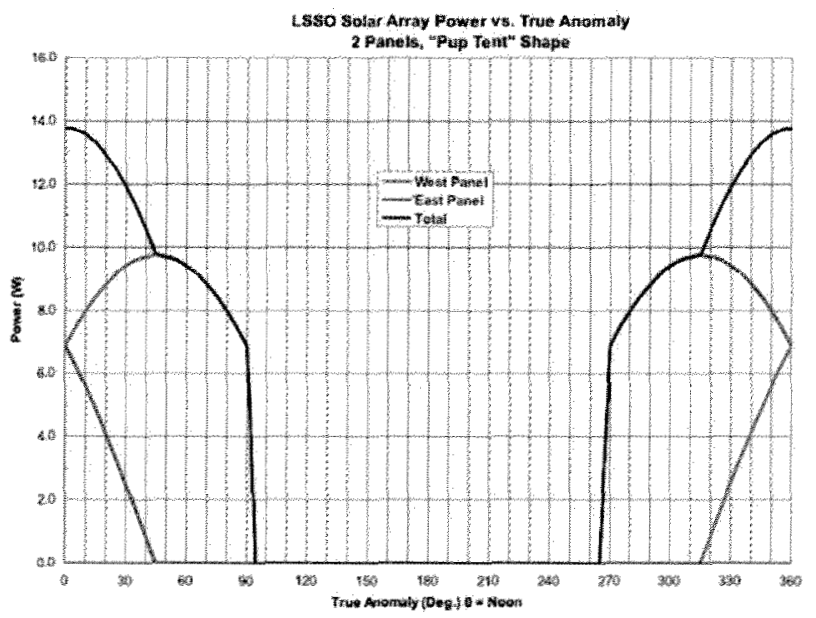

FIGURE 5. Pup-tent solar array power profile

for primary input for the CCD plane and associated electronics with a passively cooled $\mathrm{CCD}$ and $4.3 \mathrm{~W}$ primary power for a coldfire processor.

If such a design is equipped with a "pup-tent" style solar panel array $290 \mathrm{~mm}$ by $290 \mathrm{~mm}$ facing east-west to smooth out the sinusoidal variation that results from a single panel, one achieves the power profile shown in Figure 5 over the two week lunar orbit. This provides enough power during the daytime to fully charge the Lithion Model 9653 battery shown in Figure 4 which has a capacity of $1728 \mathrm{Wh}$.

Given an available $1728 \mathrm{Wh}$, at $11 \mathrm{~W}$ with no heater power, MagEX/LXO can operate for 157 hours during lunar night which is an approximately $46 \%$ duty cycle. 


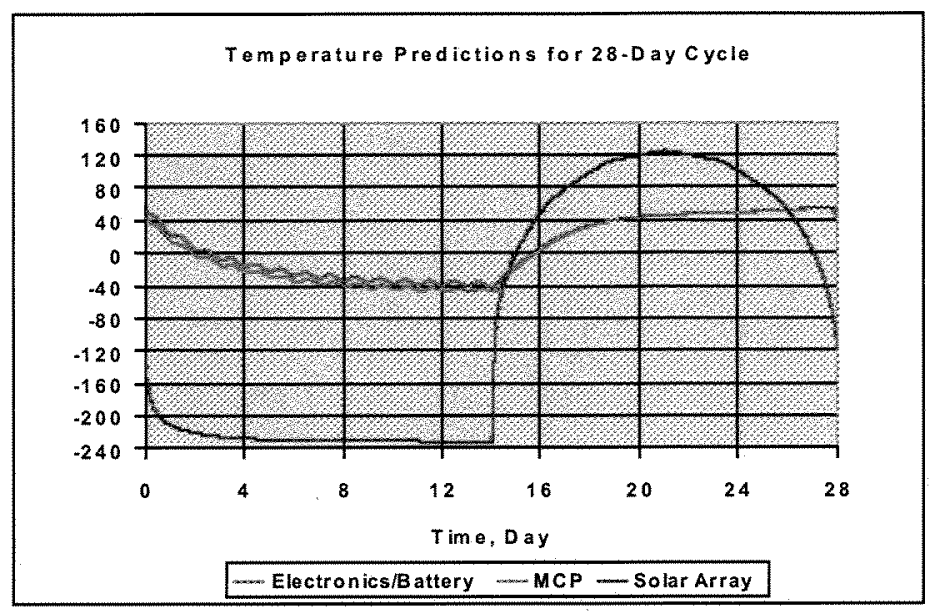

FIGURE 6. Results of the LXO/MagEX thermal analysis

\section{THERMAL CONSIDERATIONS}

One of the major considerations for the harsh lunar environment is the temperature extremes between lunar day and lunar night where the surface temperature ranges from about $-160^{\circ} \mathrm{C}$ in darkness to about $+120^{\circ} \mathrm{C}$ in sunlight, with each lasting about 14 days.

The instrument design shown in Figure 4 including battery and internal components (electronics and detectors) was modeled assuming equatorial deployment on the lunar surface. The instrument was thermally isolated through its legs from the lunar surface and the main housing as well as the back of the solar array is insulated with MLI (thermal blankets). A cover with a bimetallic actuator opening at $-80^{\circ} \mathrm{C}$ and closing at $-70^{\circ} \mathrm{C}$ to protect the detectors during daytime was modeled. The square pore slumped MCP optics element was taken to have an absorptance $\alpha=0.16$ and an emittance $\varepsilon=0.10$. The interior of the housing was modeled with high emittance, 0.90 . Radiators (white paint) with thermal louvers comprising strips around the circumference sit at the top and bottom of the upper housing. The instrument was taken to operate at $11 \mathrm{~W}$ during $46 \%$ of each 24 hour period during lunar night and was off during lunar day. The lunar surface was taken to have an emittance of 0.92 and an absorptance of 0.93 .

Figure 6 shows the results of the modeled temperature at the electronics, upper slumped MCP optics element, and solar array over a 28 -day period. The lower acceptable temperature limit for the electronics is anticipated to be about $-40 \mathrm{C}$ while the acceptable temperature range for the microchannel plate optics element, based on current knowledge, is in the range $-55 \mathrm{C}$ to $+125 \mathrm{C}$. This preliminary thermal modeling indicates that MagEX/LXO should remain above $-40 \mathrm{C}$ during night and below $+120 \mathrm{C}$ during day. 


\section{CONCLUSIONS}

No process illustrates the adage "one person's signal is another's noise" more compellingly than SWCX soft X-ray emission. Although a nuisance to astrophysicists, this process holds the potential to quite literally revolutionize heliophysics science by providing global images of the terrestrial magnetosheath and magnetopause.

\section{ACKNOWLEDGMENTS}

This material is based upon work supported by the National Aeronautics and Space Administration under Grant No. LSSO06-0032 issued through the Science Mission Directorate's Planetary Science Division.

\section{REFERENCES}

1. Cox, D.P., The Local Bubble and Beyond, Springer-Verlag: New York, 121 (1998).

2. Cravens, T.E., Geophys. Res. Lett., 24, 105-108 (1997).

3. Cravens, T.E., I.P. Robertson and S.L. Snowden, J. Geophys. Res., 106, 24,883 (2001).

4. Lisse, C.M.K. et al., Science, 274, 205-209(1996).

5. Omidi, N. and D.G. Sibeck, Geophys. Res. Lett., 34, L04016 (2007).

6. Robertson, I.P., M.R. Collier, T.E. Cravens, and M.-C. Fok, J. Geophys. Res., 111 A12, A12105 (2006).

7. Robertson, I.P. and T.E. Cravens, Geophys. Res. Lett., 30(8), 8031 (2003). 